\title{
Meningitis and CSF Rhinorrhea as Initial Presentations of Untreated Macroprolactinoma
}

\author{
Li Yunkai Andrew, Mavis Ang Kexin, Seow Cherng Jye and Lee Kim En
}

Department of Neurology, National Neuroscience Institute, Tan Tock Seng Hospital, Singapore

\begin{abstract}
Macroprolactinomas often present with hyperprolactinemia or space-occupying symptoms. There are few case reports of macroprolactinomas presenting as meningitis and CSF rhinorrhea. Presented is a case of a 51-year-old Chinese male who presented with meningitis and pneumonia, and thereafter, developed rhinorrhea. Magnetic Resonance Imaging of the brain revealed a macroprolactinoma. He subsequently underwent a trans-sphenoidal hypophysectomy to remove the tumor.
\end{abstract}

Key words: Macroprolactinoma, Meningitis, Cerebrospinal fluid rhinorhhea

\section{Introduction}

Prolactinomas are the most common secretory pituitary tumours, presenting with hyperprolactinemia including secondary amenorrhea, infertility and galactorrhea ${ }^{1}$. Some develop space-occupying symptoms including headache and bitemporal hemianopsia ${ }^{2}$. However, meningitis and cerebrospinal fluid (CSF) rhinorrhea as initial manifestations are extremely rare $^{3-4}$.

We present a patient with macroprolactinoma who presented with meningitis and CSF rhinorrhea.

\section{Case Report}

A 51-year-old gentleman, married with 3 children, presented with a 4-day history of sore throat and dry cough, associated with fever and headache. He was confused and drowsy prior to admission. Apart from a contact with flu-like symptoms, he had otherwise been well with no preceding symptoms. He had no significant past medical history.

On admission, he was agitated and febrile. Doll's reflex was present. Pupils were reactive. He moved his limbs to painful stimulus. Reflexes were normal. Bilateral crepitations were heard. Chest radiography showed bilateral consolidations. CSF nucleated cell count was 12400 cells/uL, consistent with pyogenic meningitis. Blood and CSF Cultures grew pan-sensitive Streptococcus pneumoniae. His random glucose level was $16.5 \mathrm{mmol} / \mathrm{L}$ and glycated haemoglobin was $10.5 \%$. Arterial blood gas showed high anion-gap metabolic acidosis, with betahydroxybutyrate levels of $4.2 \mathrm{mmol} / \mathrm{L}$.

He was diagnosed with disseminated pneumococcal disease, complicated by diabetic ketoacidosis. He received

ISSN 0857-1074

Copyright (C) 2011 by the JAFES

Received September 11, 2011. Accepted October 11, 2011.
2 weeks of ceftriaxone and crystalline penicillin. His blood glucose levels were closely monitored and his insulin infusion was adjusted accordingly. A Magnetic Resonance Imaging (MRI) of his head with contrast was performed to ensure no interval progression on Day 5 of admission. Instead, an incidental pituitary mass was noted (Figure 1). Prolactin level was $12867 \mathrm{ng} / \mathrm{dL}$. Apart from low testosterone levels, the other hormone levels were within normal range.

Incidentally, he subsequently complained of left nostril rhinorrhea 6 days after admission, which he previously denied having. CSF rhinorrhea was confirmed as the fluid glucose was $6.2 \mathrm{mmol} / \mathrm{L}$.

Cabergoline was started pre-operatively and he then underwent trans-sphenoidal hypophysectomy. Intraoperatively, the macroprolactinoma was found to have eroded into the sellar floor, resulting in the CSF leak. This erosion was sealed with a fat graft. He was continued on Cabergoline post-operatively as the macroprolactinoma was too vascular to remove completely.

His prolactin levels have since improved within 6 months after discharge with Cabergoline. The macroprolactinoma has remained stable in size and the patient has remained asymptomatic since discharge.

\section{Discussion}

CSF rhinorrhea is a well-known complication of dopamine agonist treatment ${ }^{1}$, trans-sphenoidal surgery and radiation. However, CSF rhinorrhea as an initial manifestation of untreated macroprolactinoma is rare. Only 21 cases have been reported since $1800 s^{4-7}$. Meningitis is an even rarer initial manifestation. Only 5 cases have

Corresponding author: Dr Li Yunkai Andrew, MBBS (Singapore), DFD (CAW) Medical Officer

National Neuroscience Institute, 11 Jalan Tan Tock Seng Singapore 308433

Tel. No.: +6597870983

Email:endrool@gmail.com 


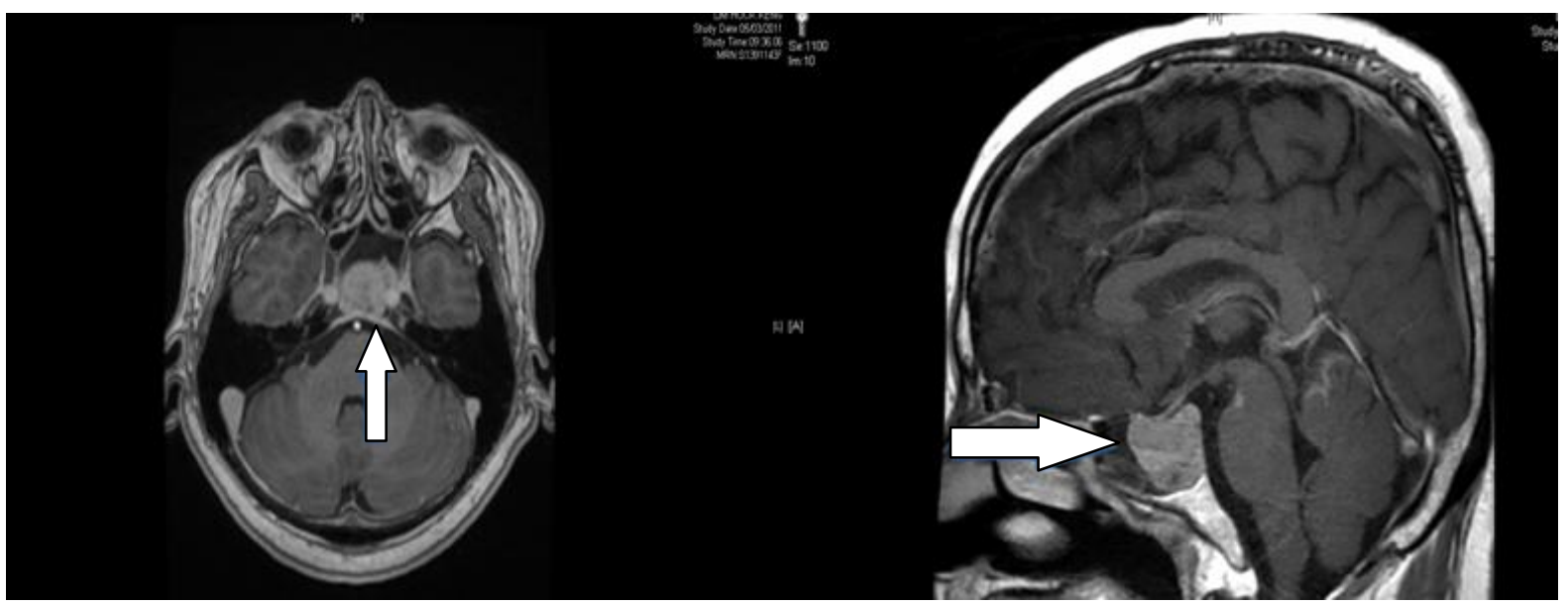

Figure 1. MRI Head with contrast shows the pituitary mass abutting both cavernous internal carotid arteries

been reported. Three cases had prior CSF rhinorrhea. One case developed CSF rhinorrhea 1 year after the meningitis episode $^{6}$. Our case report represents the second case presenting as meningitis, followed by CSF rhinorrhea.

The pathophysiology of CSF rhinorrhea in pre-treatment macroprolactinoma is unclear due to the small case numbers. Current postulations include direct extension and erosion into the sphenoid sinus, allowing fistula communication between the sella and sinuses, thereby allowing CSF leak ${ }^{7}$. Our patient's intra-operative findings were consistent with this hypothesis. Others have postulated that infarction and haemorrhage of the tumour can result in fistula formation through the tumour.

Therefore, recognition of CSF rhinorrhea is important. CSF rhinorrhea is identified by detecting Beta-2-Transferrin or rhinorrhea glucose levels of $>0.3 \mathrm{mmol} / \mathrm{L}(5 \mathrm{mg} / \mathrm{dL})$. However, rhinorrhea glucose is insensitive as external factors like meningitis can confound the result by falsely decreasing the glucose level. Our institution was unable to test for Beta-2-Transferrin, so rhinorrhea glucose was tested.

The sphenoid bone erosion was the likely cause of meningitis in our patient. However, the newly diagnosed diabetes mellitus was a confounding factor as it is known to increase a patient's susceptibility to infection. His initial flu-like symptoms, significant contact history and chest radiography findings led us to assume that he had initially developed pneumonia, which was complicated by meningitis due to his increased susceptibility to infection. We also assumed his rhinorrhea was related to his flu-like symptoms till we discovered the incidental macroprolactinoma. Our patient had not exhibited any signs of a space-occupying lesion or hyperprolactinemia. He fathered 3 children.

Medical therapy in the form of dopamine agonists serves as the first-line therapy for prolactinomas. However, the presence of a fistula mandates surgical intervention. Some advocate removing the macroprolactinoma and repairing the leak ${ }^{6}$. Others promote continued use of dopamine agonist and using muscle flaps to repair the fistula ${ }^{3}$. There is currently no consensus as such cases are too few to conduct comparison studies. We chose the former option and our patient tolerated the procedure well.

\section{Conclusion}

CSF rhinorrhea and meningitis as initial presentations of macroprolactinoma are extremely rare. This case serves to illustrate the pitfalls one may encounter and the importance of evaluating the symptoms a patient presents with, no matter how insignificant they may be.

\section{References}

1. Klibanski A. Prolactinomas. N Engl J Med. 2010; 362:1219-1226.

2. Strebel PM, Zacur HA, Gold EB. Headache, hyperprolactinemia, and prolactinomas. Obstet Gynecol. 1986 Aug; 68(2):195-199.

3. Suliman S.G.I., Gurlek A, Byrne J.V., et al. Nonsurgical Cerebrospinal Fluid Rhinorrhea in Invasive Macroprolactinoma: Incidence, Radiological and Clinicopathological features. J. Clin. Endocrinol. Metab. 2007; 92:3829-3835.

4. Cole I E. Cerebrospinal fluid rhinorrhea in pituitary tumours. J R Soc Med. 1980 April; 73(4):244-254.

5. Obana WG, Hodes JE, Weinstein PR, Wilson CB. Cerebrospinal fluid rhinorrhea in patients with untreated pituitary adenoma: Report of two cases. Surg Neurol. 1990; 33:336-340

6. Honegger J, Psaras T, Petrick M, Reincke M. Meningitis as a presentation of macroprolactinoma. Exp Clin Endocrinol Diabetes. 2009; 117:361-364

7. Ohtakara K, Matsubara T, Kojima T, Taki W, Wara S. Cerebrospinal fluid rhinorrhea associated with untreated prolactinoma: Case report. Neurol Med Chir (Tokyo). 2000; 40:413-418. 